\title{
Signatures of GMSB with non-pointing photons at the ATLAS Detector
}

\section{Helen Hayward on behalf of the ATLAS collaboration SUSY09 5-10 June}




\section{Outline}

-GMSB Theory

-ATLAS LArCalorimeter

-Signature of Non-pointing photons

-Photon Reconstruction

-Photon Identification

-Event Selection

-Extraction of Neutralino lifetime

- using photon direction

- using calorimeter timing

-Conclusion 


\section{Theory}

-SUSY is good candidate for physics beyond the SM

typical decay chain for Neutralino NLSP

-In GMSB, the symmetry is broken by gauge interactions through messenger gauge fields.

\begin{tabular}{|l|l|}
\hline$\Lambda$ & SUSY Breaking Scale \\
\hline $\mathrm{M}$ & Messenger Mass Scale \\
\hline $\tan \beta$ & Ratio of Higgs VEVs \\
\hline $\mathrm{N}$ & Number of Higgs mass parameter \\
\hline $\operatorname{sign}(\mu)$ & Sign of Higgs mass parameter \\
\hline $\mathrm{C}_{\text {grav }}$ & Scale factor of Gravitino coupling \\
\hline
\end{tabular}

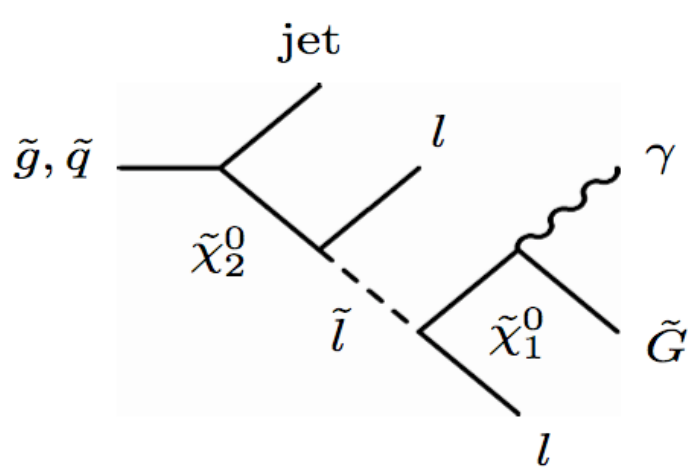

-if $N=1, \tan \beta$ is low, the NLSP is $\widetilde{\chi_{0}^{1}}$

-BR of neutralino to Gravitino plus photon is $97 \%$

$N_{5}=1, \tan \beta=5, \operatorname{sgn}(\mu)=+$

\begin{tabular}{|c|c|c|c|c|c|c|}
\hline name & NLO (LO) $\sigma[\mathrm{pb}]$ & $\Lambda[\mathrm{TeV}]$ & $M_{m}[\mathrm{TeV}]$ & $C_{G}$ & $c \tau[\mathrm{mm}]$ & $M_{\tilde{\chi}_{1}^{0}}[\mathrm{GeV}]$ \\
\hline GMSB1 & $7.8(5.1)$ & 90 & 500 & 1.0 & 1.1 & 118.8 \\
\hline GMSB2 & $7.8(5.1)$ & 90 & 500 & 30.0 & $9.5 \cdot 10^{2}$ & 118.8 \\
\hline GMSB3 & $7.8(5.1)$ & 90 & 500 & 55.0 & $3.2 \cdot 10^{3}$ & 118.8 \\
\hline
\end{tabular}




\section{Non-pointing photons}

- In certain GMSB scenarios the $\widetilde{\chi_{0}^{1}}$ could be relatively long-lived.

- If decay length is comparable to the size of the ATLAS inner detector, high $\mathrm{pT}$ photons could enter the calorimeter at angles $(\eta \curlyvee)$ deviating significantly from the nominal pointing angle $\left(\eta_{2}\right)$.

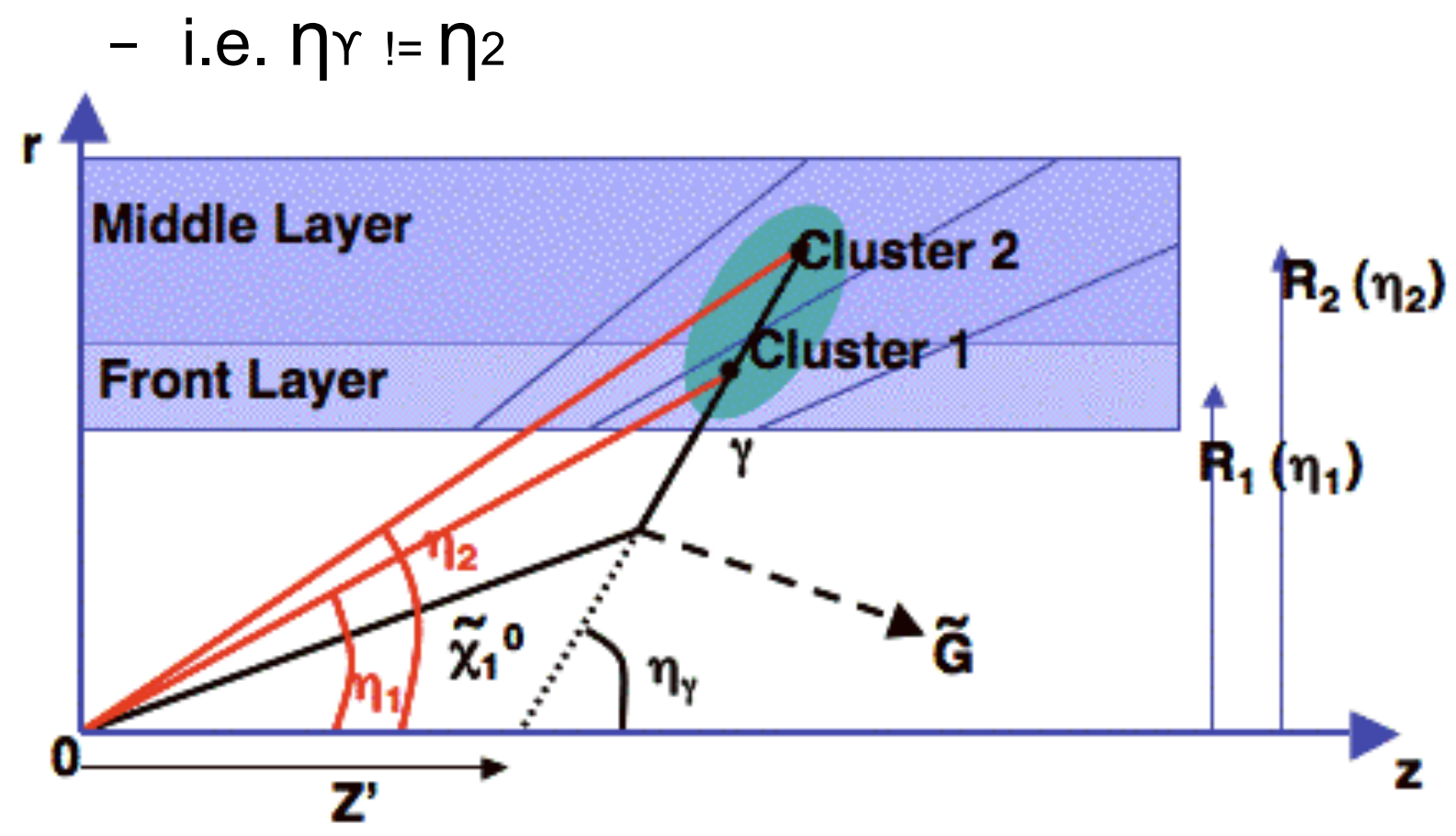

define:

- $\eta_{2}$ as "detector eta" of photon

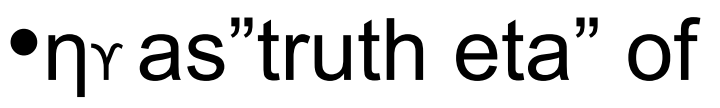
photon

( $\eta r$ is corrected for z-co-ordinate of primary vertex) Helen Hayward, ATLAS Collaboration - SUSY09 


\section{ATLAS Detector}

- Tracking extends to $|\eta|=2.5$

- Pixel, SemiConductor and Transition Radiation Trackers

- Calorimeter:

- Liquid Argon EM Calorimeter (Lar EM)

- Tile Calorimeter

- barrel calorimeter extends to:

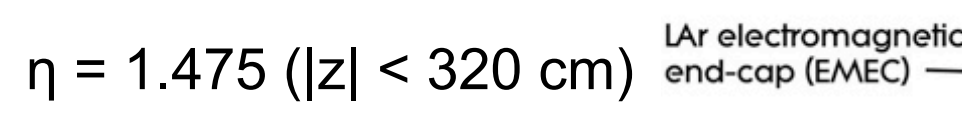

- endcap calorimeter consists of two coaxial wheels covering $1.375<|\eta|<2.5$ and $2.5<|\eta|<3.2$

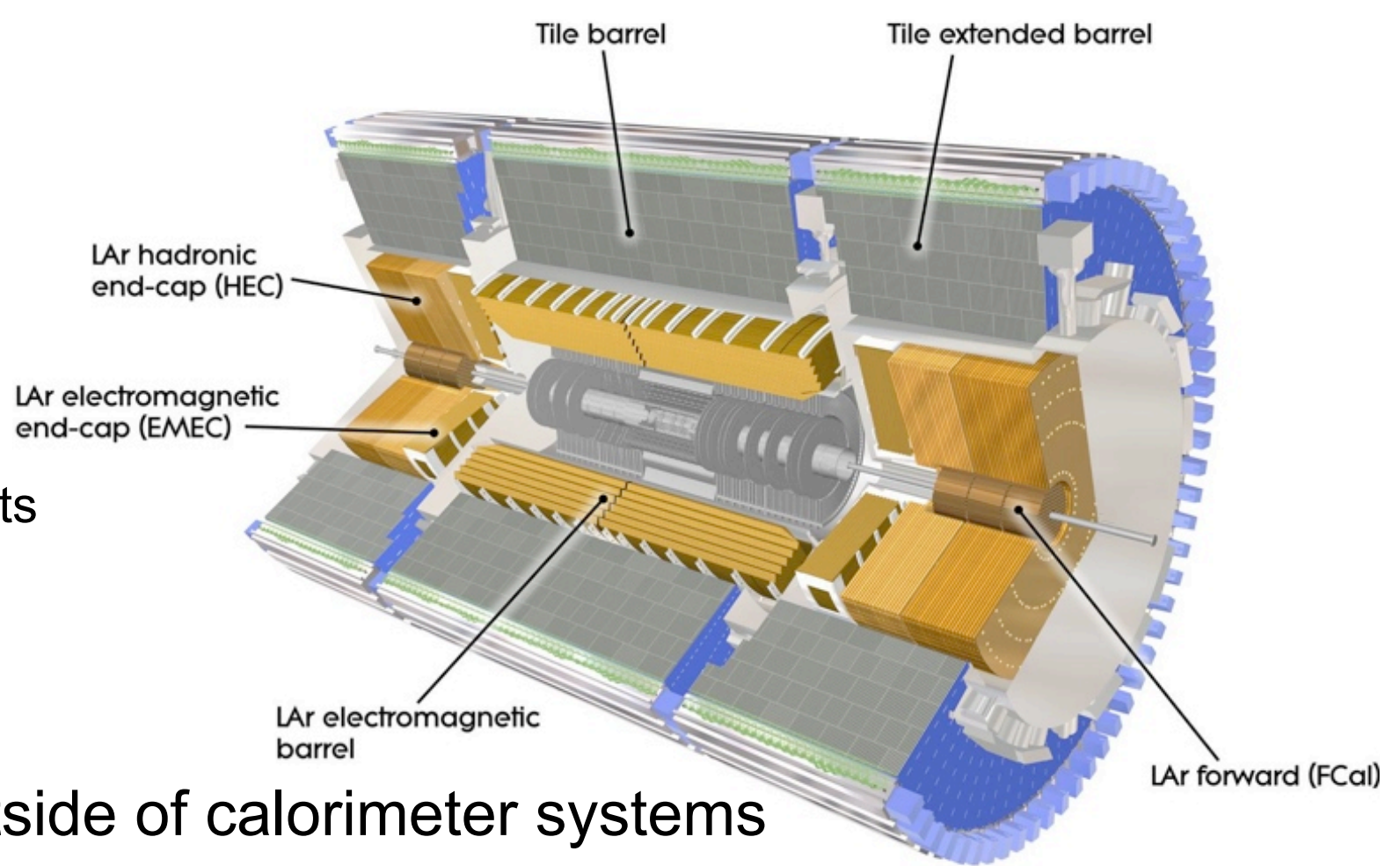

- Muon chambers sit outside of calorimeter systems

Please see talks by Olivier Arnaez and Mauro Donega on the egamma performance and reconstruction / identification for more details. 


\section{LAr EM Calorimeter}

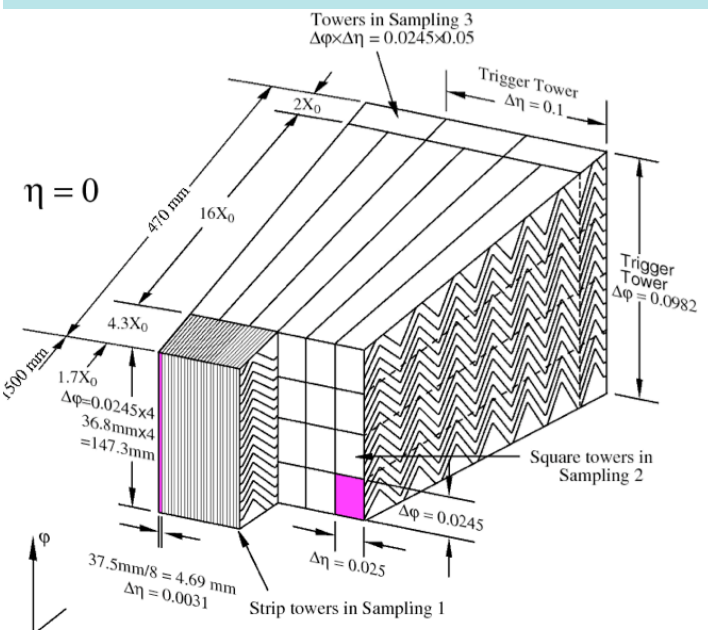

Moliere radius in liquid argon is $10.1 \mathrm{~cm}$.

Typical width for $40 \mathrm{GeV}$ shower of unconverted pointing photon, at $\eta=0.3$ is $15.5 \mathrm{mrad}(\sim 2.5 \mathrm{~cm})$

June !
- fine segmentation in 1st sampling devoted to pi0/ gamma separation in eta.

- accordion structure to reduce gaps

- towers "point" to nominal interaction point

- timing capability

- for $\mathrm{E}>1 \mathrm{GeV}$, in barrel:

- $\sigma_{\text {time }}<1 \mathrm{~ns}$

- directional resolution

- shower barycentre in $\eta$ is measured in 2 positions in depth; i.e. in 1st and 2nd sampling.

- $\sigma_{\vartheta} \bullet \sqrt{ } \mathrm{E}<75(\mathrm{mrad} \bullet \sqrt{\mathrm{GeV}})$

- (excluding crack region between barrel and endcap calorimeters) 


\section{Photon Reconstruction}

- a "sliding window" clustering algorithm is used to find showers produced by electrons and photons in the LAr calorimeter.

- Cells are clustered in $3 \times 7$ fixed size rectangles $(\eta \times \phi)$.

- Position of rectangle/window is adjusted so that it contains transverse energy at a local maximum

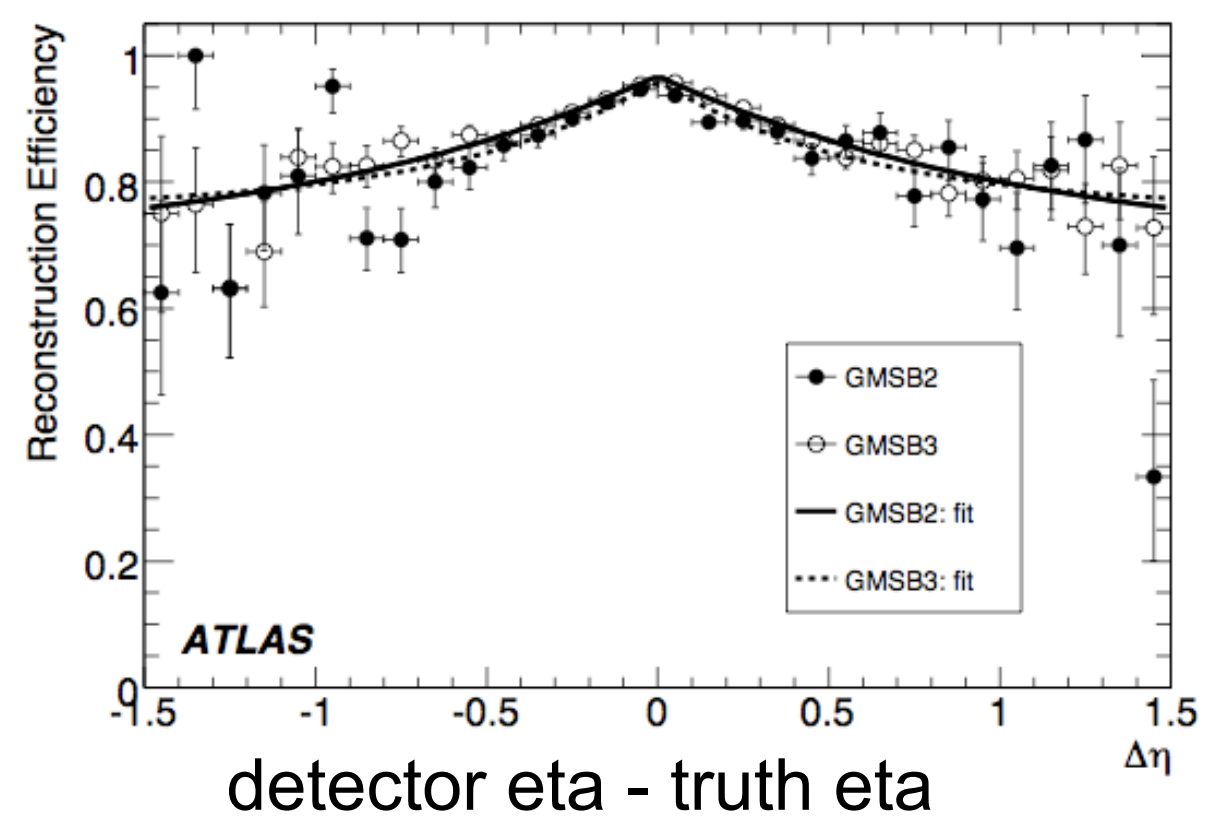

- Photons used:

- originate from neutralino decay occurring inside volume of inner detector

- pT > $20 \mathrm{GeV}$

- $\mid$ detector eta $\mid<2.5$

- match if within $\Delta \mathrm{R}=0.2$ of truth photon

As photons become more "nonpointing, the energy can be deposited in more cells.

This reduces the chance that the sliding window algorithm can correctly identify the photon.

Work ongoing to optimize clustering and photon reconstruction for nonpointing photons 


\section{Photon Identification}

- Photon Id is based on variables describing shape of energy deposit.

- non-pointing photons can have a wider shower profile than pointing photons

- Variables based on shower width highly biased w.r.t. photons produced from long-lived neutralino.

- Use only photon id selection that are unbiased w.r.t neutralino decay length

e.g: ratio of energy in $3 \times 7$ to $7 \times 7$ window in 2 nd sampling layer

width of cluster in second sampling
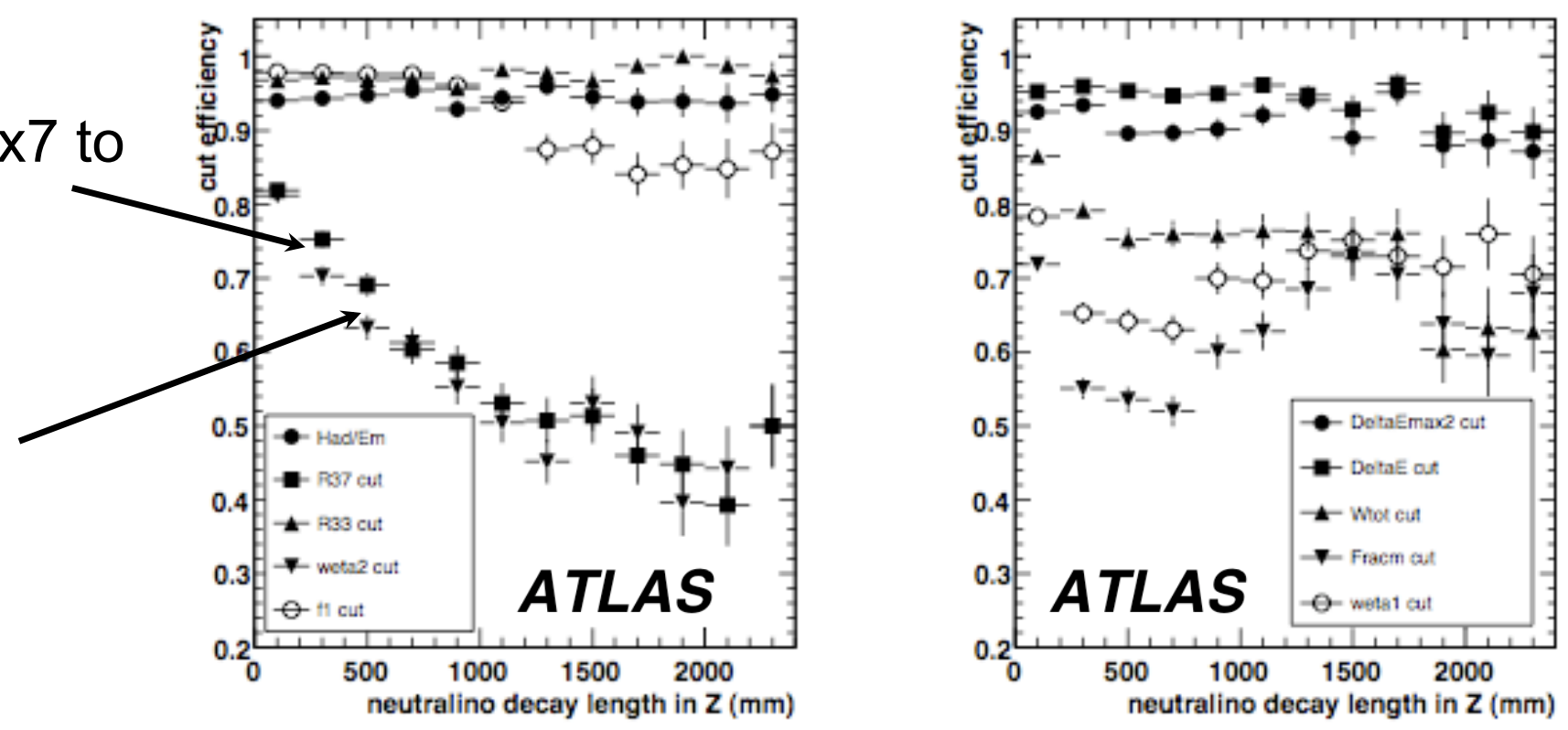

The photon Id cuts can be loosened, in order to be unbiased, at the cost of increasing the fraction of jets identified as photons from $0.19+/-0.03 \%$ to $0.70+/-0.07 \%$ 


\section{Event Selection: Trigger}

- 2 strategies considered

- Trigger directly on photon(s)

- Trigger on jets (and missing Et)

- Standard photons triggers are inefficient for non-pointing photons

- Can successfully trigger on jets (and missing $\mathrm{E}_{\mathrm{T}}$ triggers)

\begin{tabular}{|llll|}
\hline Trigger & $\mathrm{L} 1$ & $\mathrm{~L} 1+\mathrm{L} 2$ & $\mathrm{~L} 1+\mathrm{L} 2+\mathrm{EF}$ \\
\hline Single $\mathrm{Y}\left(\mathrm{E}_{\mathrm{T}}>55 \mathrm{GeV}\right)$ & $90.19 \pm 1.08$ & $46.04 \pm 1.81$ & $36.88 \pm 1.75$ \\
\hline Two $\mathrm{Y}\left(\mathrm{E}_{\mathrm{T}}>17 \mathrm{GeV}\right)$ & $34.13 \pm 1.72$ & $17.77 \pm 1.39$ & $12.87 \pm 1.22$ \\
\hline $\begin{array}{l}\text { One jet }\left(\mathrm{E}_{\mathrm{T}}>65 \mathrm{GeV}\right) \text { plus } \\
\text { missing } \mathrm{E}_{\mathrm{T}}>70 \mathrm{GeV}\end{array}$ & $80.38 \pm 0.56$ & $80.24 \pm 0.56$ & $71.81 \pm 0.64$ \\
\hline Three jets $\left(\mathrm{E}_{\mathrm{T}}>65 \mathrm{GeV}\right.$ & $79.80 \pm 0.57$ & $79.66 \pm 0.57$ & $79.62 \pm 0.57$ \\
\hline
\end{tabular}

Trigger efficiency for GMSB3 sample (generated mean lifetime $10.7 \mathrm{~ns}$ ) and $\mathcal{L}=10^{33} \mathrm{~cm}^{-2} \mathrm{~s}^{-1}$ 


\section{Event Selection}

- Require $\mathrm{N}_{\text {jets }} \geq 4$ with $p_{\mathrm{T}}>50 \mathrm{GeV}$

- Require leading jet $p_{T}>100 \mathrm{GeV}$

- Missing $E_{T}>100 \mathrm{GeV}$

- Missing $E_{T}>0.2 \times M_{\text {eff }}$

\section{Integrated}

luminosity $=$

$$
1 f b^{-1}
$$

- Where $M_{\text {eff }}$ is scalar sum of Missing $E_{T}$ and $p_{T}$ of 4 leading jets

\begin{tabular}{|r|r||r|r|r||r|r|r|}
\hline $\mathrm{N}_{\gamma}$ & $\mathrm{N}_{\text {OSSF }}$ & Signal & Background & $\mathrm{Sig}$ & $\mathrm{N}_{W}$ & $\mathrm{~N}_{Z}$ & $\mathrm{~N}_{t \bar{t}}$ \\
\hline 0 & 0 & 825.2 & 929.6 & 27.1 & 274.4 & 21.0 & 632.8 \\
0 & 1 & 265.2 & 73.0 & 33.2 & 8.7 & 1.4 & 63.0 \\
1 & 0 & 255.8 & 51.7 & 35.7 & 19.5 & 2.0 & 30.1 \\
1 & 1 & 68.6 & 1.4 & 58.6 & 0.2 & 0.0 & 1.2 \\
2 & 0 & 12.5 & 0.1 & 12.5 & 0.0 & 0.0 & 0.1 \\
2 & 1 & 4.7 & 0.0 & 4.7 & 0.0 & 0.0 & 0.0 \\
\hline
\end{tabular}

- $t \bar{t}$ is major source of background

- Event selection can be optimised by requiring 1 photon and 1 oppositesign-same-flavour(OSSF) pair of leptons. 


\section{GMSB Events}

Distributions show signal and SM background distributions before and after requirements on leptons and photons in the event.

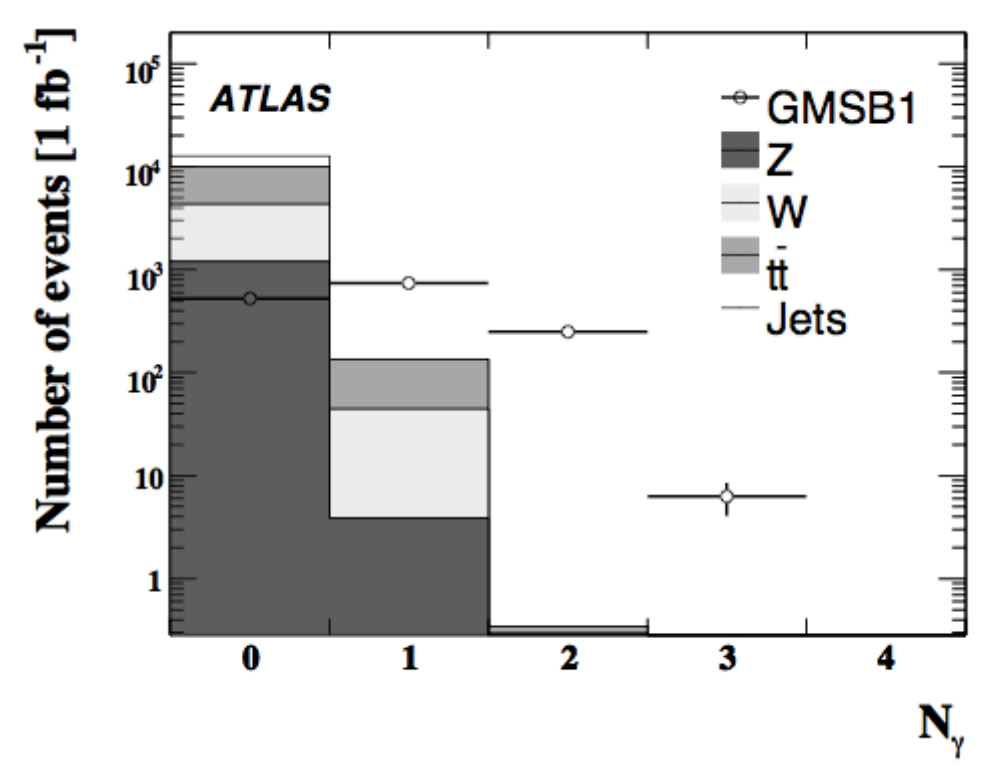

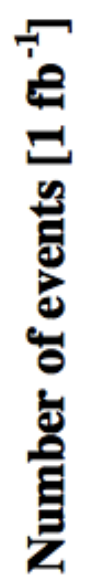
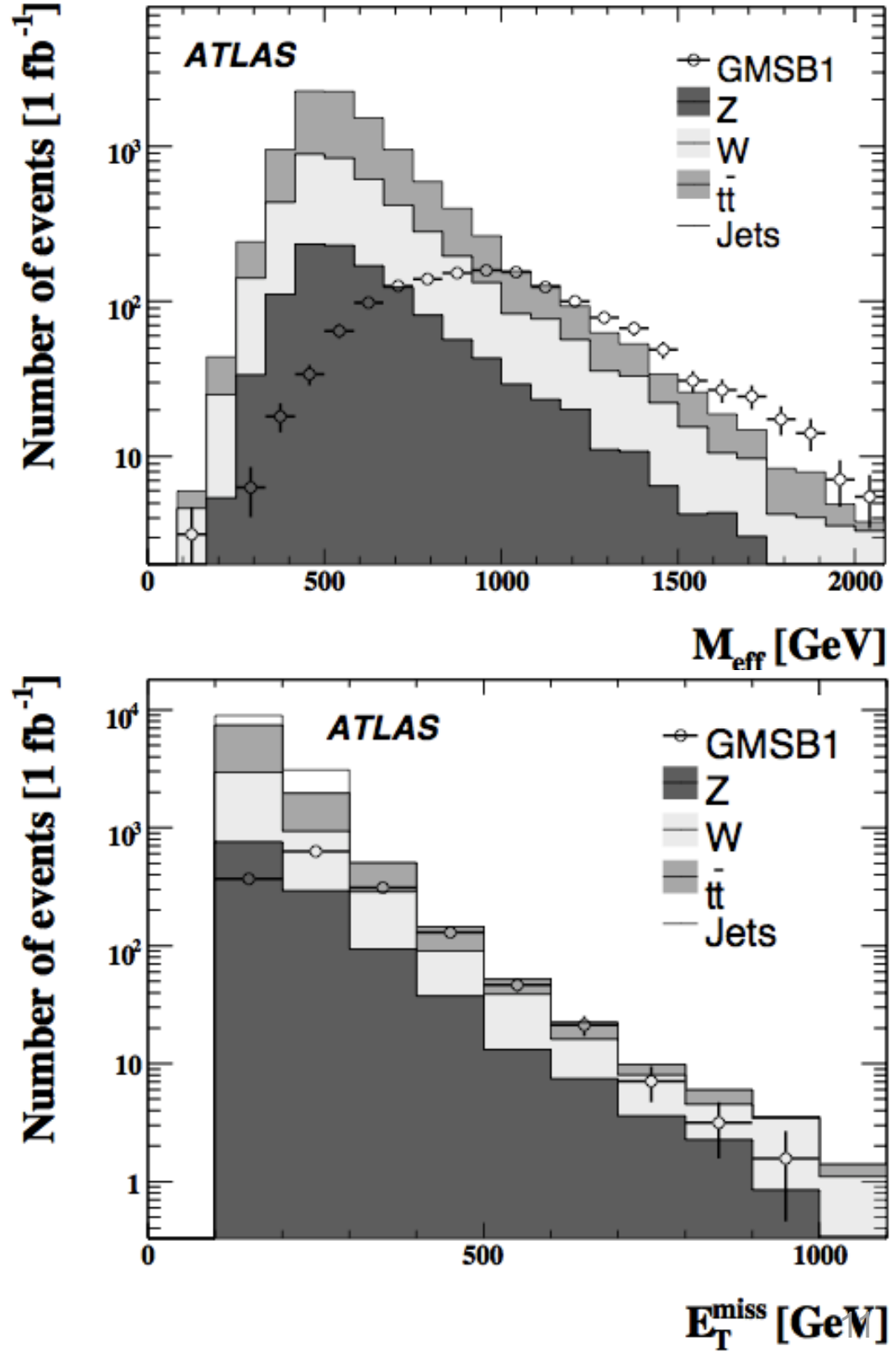


\section{Extracting lifetime: \\ Projected Longitudinal Impact Parameter}

- Define Z' as the photon's projected longitudinal impact parameter

- The Z' distribution is plotted for all photons

- (corrected for vertex displacement)

- exponential function is fitted from $50 \mathrm{~mm}$ to $500 \mathrm{~mm}$

- to exclude vertex effects

- to ensure decay occurs inside volume of inner detector

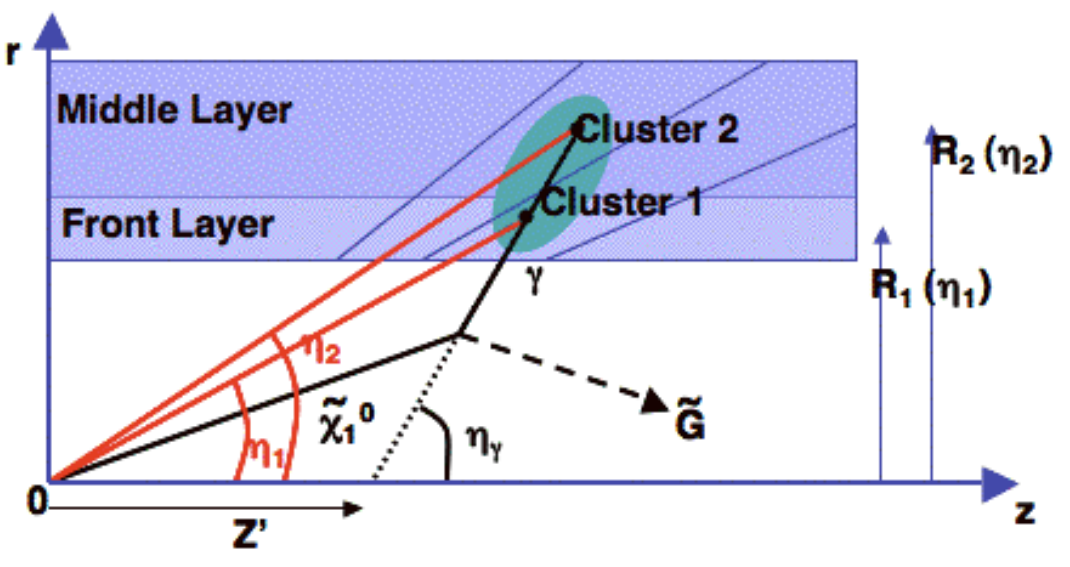

Record slope of exponential:

GMSB2: $-4.35(6) \times 10^{-3}$

$$
\text { GMSB3 : }-3.8(2) \times 10^{-3}
$$

- (sys error due to Reconstruction efficiency $=0.05 \times 10^{-3}$ )

- GMSB2: Generated $\mathrm{T}=3.17 \mathrm{~ns}$

- Slope $=-4.35(6) \times 10^{-3}$

- GMSB3: Generated $\mathrm{T}=10.7 \mathrm{~ns}$

- Slope $=-3.8(2) \times 10^{-3}$ 


\section{Extracting lifetime: Projected Longitudinal Impact Parameter}

- Need a calibration curve to relate slope back to lifetime.

- Need full simulation of many lifetime samples for accurate determination of calibration curve

Example calibration plot, produced from Toy MC

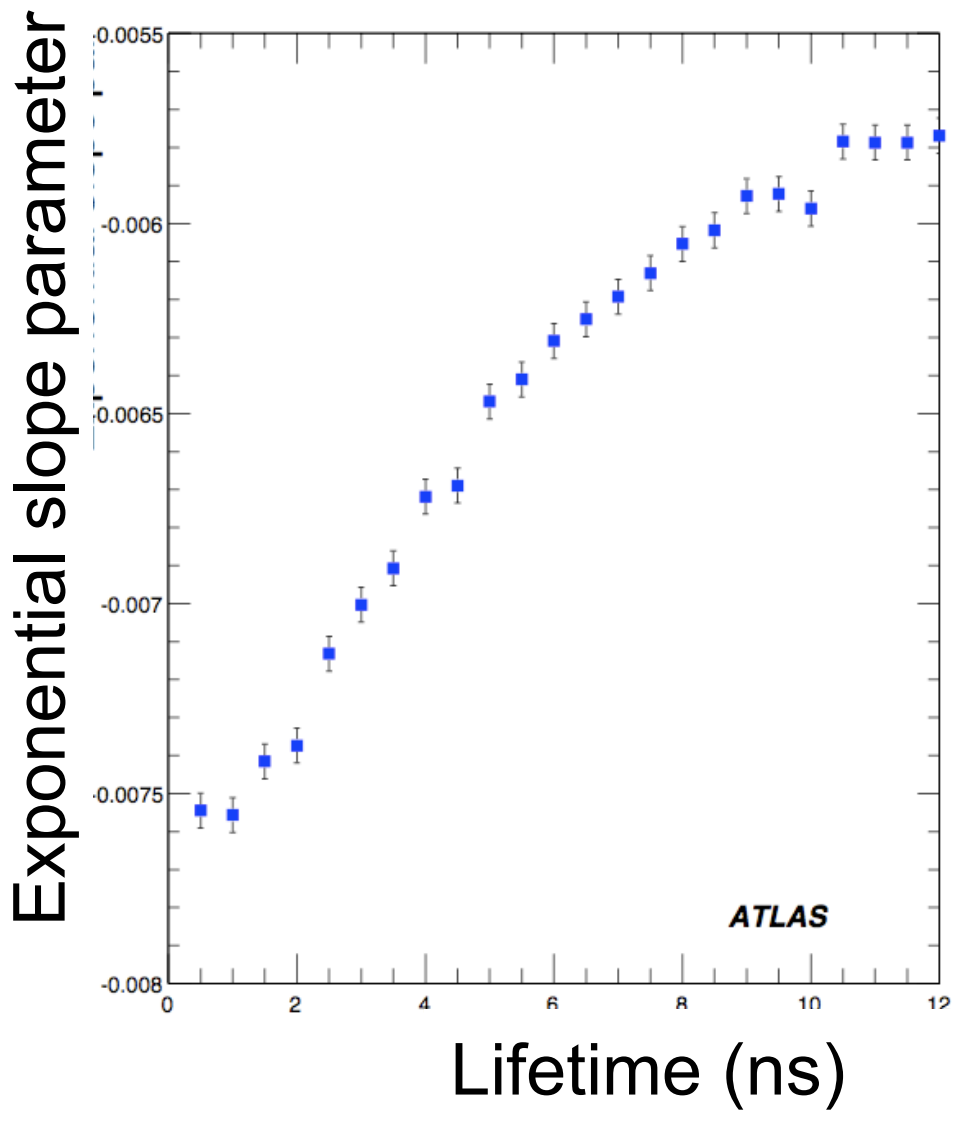




\section{Extracting lifetime: Timing (I)}

cluster time:

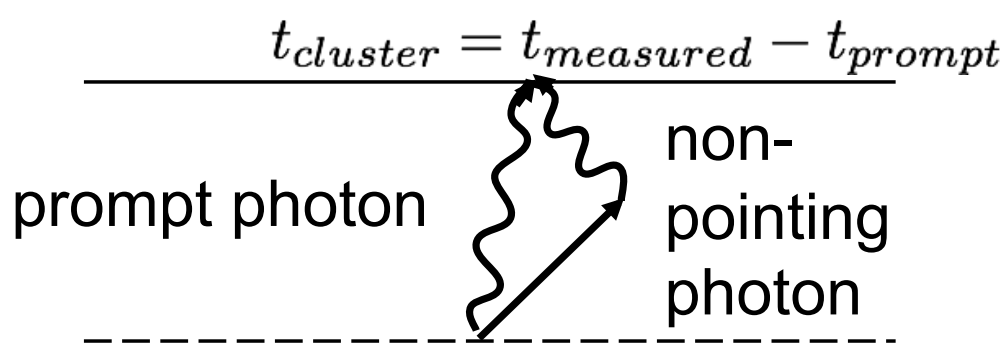

- MC truth information to calibration plot to directly relate cluster time to lifetime of neutralino.

- plot extracted neutralino lifetime distribution

-
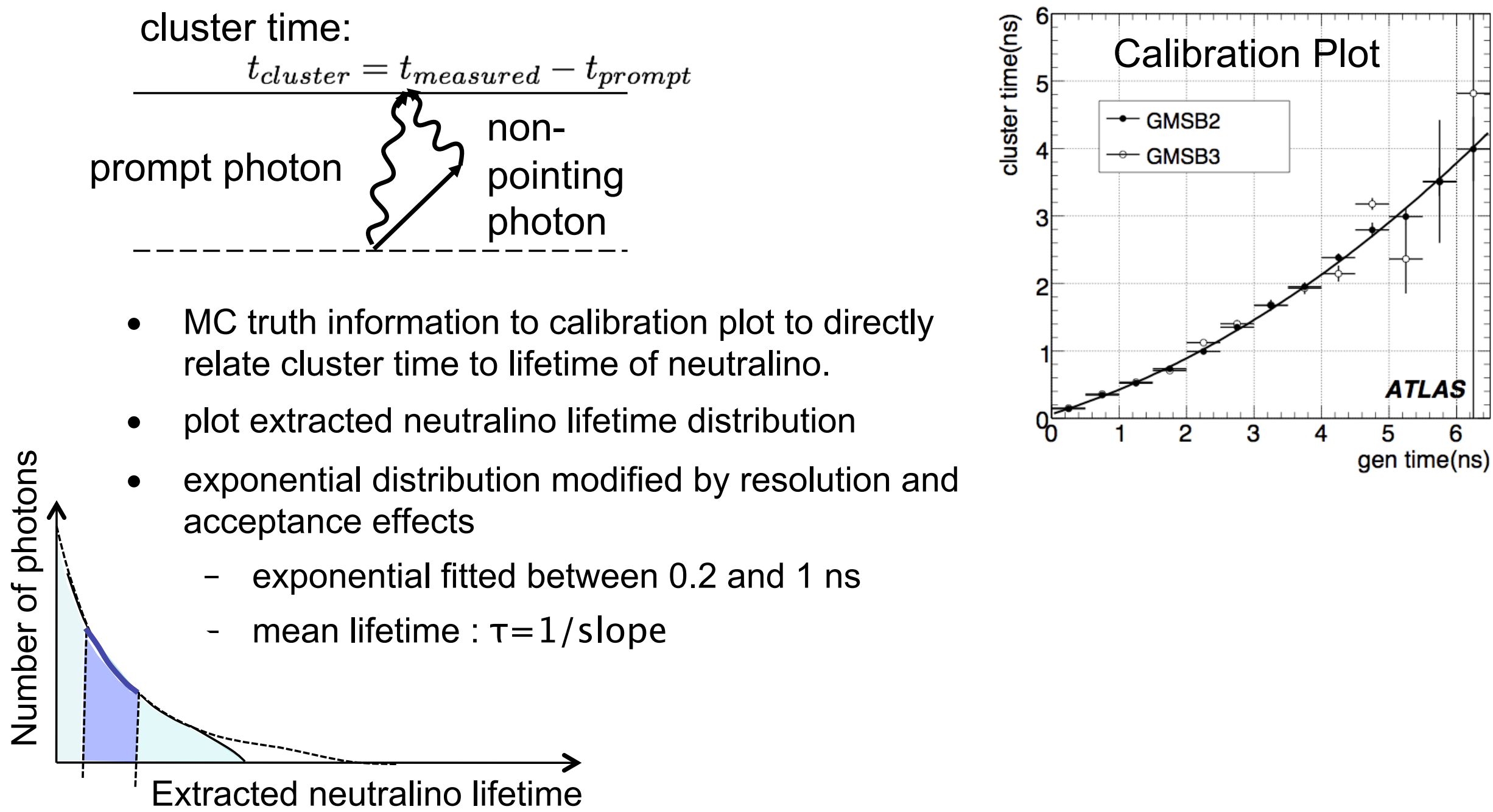


\section{Extracting lifetime: Timing (II)}

- GMSB2 (Generated mean lifetime of $3.17 \mathrm{~ns}$ )

- No photon selection: $2.9+/-0.2$ ns

- full unbiased photon selection: $3.0+/-0.2 \mathrm{~ns}$

- GMSB3 (Generated mean lifetime of $10.7 \mathrm{~ns}$ )

- No photon selection: 9 +/- 4 ns

- full unbiased photon selection: 19 +/- 19 ns

- Any dependence of photon id efficiency on neutralino lifetime biases the result

- large errors for GMSB3 sample due to lack of statistics over limited fitting range

- sys error 1(10) ns for GMSB2 (GMSB3) sample. 


\section{Summary}

- GMSB SUSY has signature similar to standard MSSM SUSY, plus two high $\mathrm{p}_{\mathrm{T}}$ photons.

- The ATLAS calorimeters have been designed with good timing and directional resolution

- Non-pointing photons provide unique signature

- Techniques are being developed to optimize detection of non-pointing photons at ATLAS

- Techniques are being developed to extract lifetime of neutralino using timing and directional information from $\mathrm{LAr}$ Calorimeter

- Future work to assess the use of photon conversions which should give useful cross-check of lifetime measurement 